\title{
Magnetohydrodynamic Spectral Theory of Laboratory and Astrophysical Plasmas
}

\author{
Hans Goedbloed ${ }^{\text {*t }}$ \\ FOM-Institute for Plasma Physics 'Rijnhuizen' \\ \& Astronomical Institute, Utrecht University, the Netherlands \\ E-mail: goedbloed@rijnh.nl \\ Home page: http://www.rijnh.nl/users/goedbloed
}

Spectral theory of linear waves and instabilities of magnetohydrodynamics (MHD) describes an enormous variety of plasma dynamics in the laboratory and in the Universe. The reason is the abundance of plasma: more than $90 \%$ of visible matter in the Universe is plasma, whereas dark matter may have a substantial plasma component as well. The theoretical basis comes from the fact that the MHD equations are scale-independent and that MHD spectral theory can be cast in a completely analogous form to that of quantum mechanics: the MHD force operator is self-adjoint in the Hilbert space of plasma displacement vectors [1]. Nevertheless, MHD spectral theory is still very incomplete at present. In particular, group theoretical investigation of symmetry properties has hardly been undertaken. Considering the central role plasmas are to play in a future model of the Universe, this calls for a major mathematical effort.

Probably, the complicating factor is the omni-presence of large background flows, which are often super'sonic' (surpassing one the three critical MHD speeds), implying that the standard picture developed for static plasmas breaks down: Plasma dynamics is to be described by non-selfadjoint operators and the necessary background states are frequently transonic. The mathematical problems associated with these two features are enormous, but recently found monotonicity properties of the complex spectrum and singularities in the equilibrium flows provide confidence that a meaningful structure will eventually emerge.

This will be illustrated with recent results on the spectrum of accretion disks [2] and new instabilities driven by transonic transitions of the flow that involve singular trans-slow Alfvén modes with a continuous spectrum [3]. Except that these instabilities appear to have exciting physical properties (facilitating accretion flows and jet formation), they have quite interesting geometrical properties as well since they 'live' on the curved two- dimensional surfaces spanned by the plasma velocity and magnetic fields.

Fifth International Conference on Mathematical Methods in Physics - IC2006

Centro Brasileiro de Pesquisas Fisicas, Rio de Janeiro, Brazil

24-28 April, 2006

\footnotetext{
* Speaker.

${ }^{\dagger}$ Fruitful collaboration with Rony Keppens is acknowledged. This work was performed as part of the research program of the Euratom-FOM Association Agreement, with support from the Netherlands Science Organization (NWO).
} 


\section{Introduction}

Astronomical observations show that plasma is the most abundant ( $>90 \%)$ state of matter in the Universe. Recently, dark matter is also considered to have a substantial plasma component. Hence, on should expect plasmas to play a major role in future cosmology. A crude definition reads: plasma is a completely ionized gas of freely moving positively charged nuclei and negatively charged electrons. On Earth this state of matter is exceptional, obtained in laboratory thermonuclear fusion experiments at high temperatures $\left(T \sim 10^{8} \mathrm{~K}\right)$. In astrophysics, plasmas are ever more prominent (stellar coronae, magnetospheres, pulsars, BH accretion disks, AGN jets, etc.).

What is so special about plasma? Plasma is essentially a global state because of the embedded magnetic field, so that it is meaningless to discuss an isolated small piece of plasma.

Consider the standard view of nature (Fig. 1). In between elementary particles interacting through the nuclear forces and astronomical objects kept together by the gravitational force, the intermediate levels are dominated by the electrostatic force giving rise to atoms and molecules, the electrically neutral building blocks of ordinary matter. The electrostatic force is 'exhausted' on the larger distance scales: the dots in the figure appear to indicate that nothing of fundamental interest happens over 18 decades in length scale!

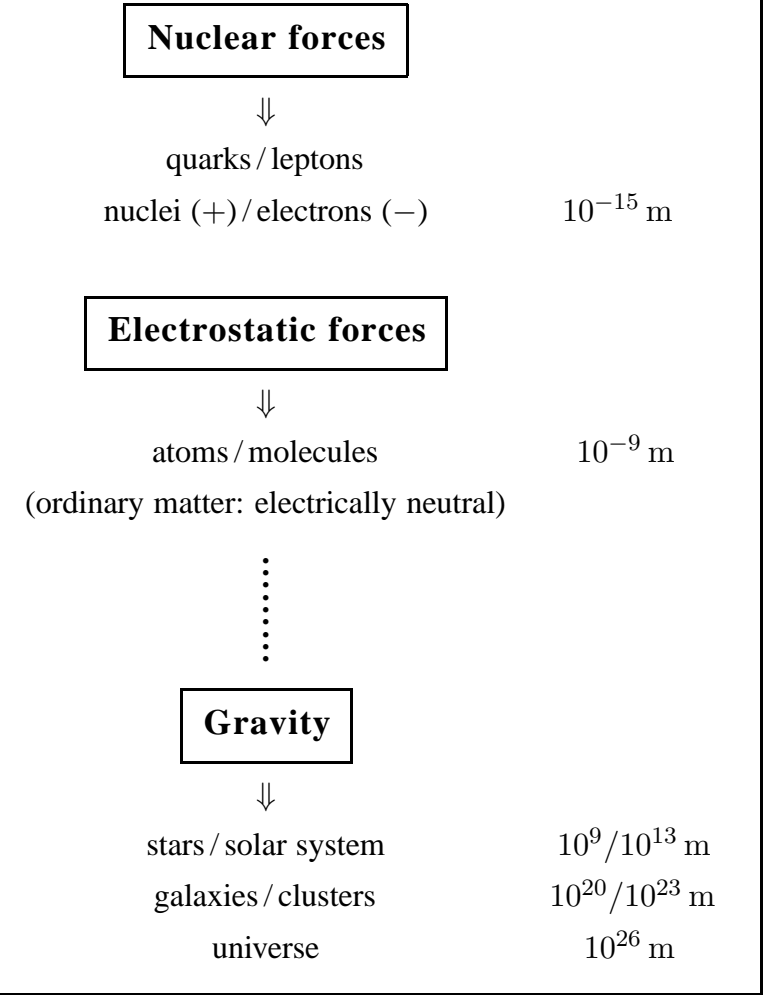

Figure 1: Standard view of nature

The mistake implicit in this figure is that the main constituent of the Universe is assumed to be ordinary matter instead of plasma. Plasma is also electrically neutral, but the nuclei $(+)$ and electrons (-) are not tied in atoms or molecules but freely move about to form one collective fluid. The unavoidable result is induction of electric currents and magnetic fields, giving rise to large- 
scale plasma dynamics determined by magnetic flux conservation. In contrast to the omni-present spherical symmetry of electrostatic and gravitational forces in atomic physics and astronomy, such symmetries are absent on the plasma scale: $\nabla \cdot \mathbf{B}=0$ is incompatible with spherical symmetry (evidenced by the violent disruptions of stellar magnetic configurations). Instead, the magnetic plasma confinement geometries (toroidal or infinitely long tubular structures) become the basic entities. Hence, magnetohydrodynamics (MHD) describes the intermediate levels of the Universe.

Theoretical plasma models roughly fall into three categories:

(1) Kinetic theories, describing the local small scales by means of Boltzmann equations for the electron and ion distribution functions $f_{e, i}(\mathbf{r}, \mathbf{v}, t)$, coupled with Maxwell's equations for the electric and magnetic fields $\mathbf{E}, \mathbf{B}(\mathbf{r}, t)$;

(2) Two-fluid theories, describing both small and large scales by means of electron and ion fluid variables $n_{e, i}, \mathbf{u}_{e, i}, p_{e, i}(\mathbf{r}, t)$, again coupled with Maxwell's equations for $\mathbf{E}, \mathbf{B}(\mathbf{r}, t)$;

(3) Magnetohydrodynamics, describing global large scales by means of the single fluid variables $\rho$, $\mathbf{v}, p, \mathbf{B}(\mathbf{r}, t)$. Frequently, dissipation is a slow process so that dissipative MHD simplifies to ideal MHD, the simplest and most effective of all plasma models.

\begin{tabular}{llll}
\hline \hline & $l_{0}(\mathrm{~m})$ & $B_{0}(\mathrm{~T})$ & $t_{0}(\mathrm{~s})$ \\
\hline tokamak & 20 & 3 & $3 \times 10^{-6}$ \\
magnetosphere Earth & $4 \times 10^{7}$ & $3 \times 10^{-5}$ & 6 \\
solar coronal loop & $10^{8}$ & $3 \times 10^{-2}$ & 15 \\
magnetosphere neutron star & $10^{6}$ & $10^{8 *}$ & $10^{-2}$ \\
accretion disc YSO & $1.5 \times 10^{9}$ & $10^{-4}$ & $7 \times 10^{5}$ \\
accretion disc AGN & $4 \times 10^{18}$ & $10^{-4}$ & $2 \times 10^{12}$ \\
galactic plasma & $10^{21}$ & $10^{-8}$ & $10^{15}$ \\
& $\left(=10^{5} \mathrm{ly}\right)$ & & $\left(=3 \times 10^{7} \mathrm{y}\right)$ \\
\hline \hline
\end{tabular}

Table 1: Scales of actual plasmas

The ideal MHD equations are obtained by averaging the kinetic equations over space and time, breaking the symmetry between $\mathbf{E}$ and $\mathbf{B}$ by exploiting pre-Maxwell's equations (neglecting displacement current and electrostatic force), and determining the electric field from $\mathbf{E}=-\mathbf{v} \times \mathbf{B}$ :

$$
\begin{aligned}
& \frac{\partial \rho}{\partial t}+\nabla \cdot(\rho \mathbf{v})=0 \\
& \rho\left(\frac{\partial \mathbf{v}}{\partial t}+\mathbf{v} \cdot \nabla \mathbf{v}\right)+\nabla p+\rho \nabla \Phi-\frac{1}{\mu_{0}}(\nabla \times \mathbf{B}) \times \mathbf{B}=0, \quad \text { (momentum) } \\
& \frac{\partial p}{\partial t}+\mathbf{v} \cdot \nabla p+\gamma p \nabla \cdot \mathbf{v}=0 \\
& \frac{\partial \mathbf{B}}{\partial t}-\nabla \times(\mathbf{v} \times \mathbf{B})=0, \quad \nabla \cdot \mathbf{B}=0 . \quad \quad \text { (magnetic flux) }
\end{aligned}
$$

These equations describe an enormous variety of different plasmas (Table 1$)$ because they are scaleindependent: by proper scaling, plasma size $\left(l_{0}\right)$, magnetic field strength $\left(B_{0}\right)$, and time scale $\left(t_{0}\right)$ 
drop out of the equations. The time scale is associated with the plasma density $\left(\rho_{0}\right)$ through the Alfvén speed $v_{\mathrm{A}} \equiv B_{0} / \sqrt{\mu_{0} \rho_{0}} \Rightarrow t_{0} \equiv l_{0} / v_{\mathrm{A}}$.

To solve the MHD equations (1.1)-(1.4), two general approaches exist in plasma dynamics: (a) Keep the full nonlinear model. This involves numerical, large-scale computing (inaccurate with respect to the distinction between dynamics parallel and perpendicular to the magnetic surfaces); (b) Split in nonlinear equilibrium and linear perturbations. This leads to spectral theory, which is followed here (restricted to small amplitudes, but involving powerful mathematics).

Our angle on the field is dictated by the attempt to unify the laboratory and astrophysical pictures of MHD waves and instabilities by exploiting the scale-independence of the MHD equations. We call this MHD spectroscopy. It originated from MHD spectral theory and large-scale numerical computations applied to laboratory fusion experiments by many authors since the 1970s. MHD spectroscopy for tokamaks was proposed in Ref. [4] by following the example of helio-seismology as a very accurate method to determine the interior characteristics of the Sun. A similar, astrophysical, program is magneto-seismology of accretion disks about compact objects [2].

\section{MHD spectral theory of static plasmas}

For static plasmas (no background velocities), linearization proceeds according to the scheme: (a) Static equilibrium variables $\rho_{0}, p_{0}, \mathbf{B}_{0}$ (functions of $\mathbf{r}$ ) are determined by the nonlinear PDEs

$$
\mathbf{j}_{0} \times \mathbf{B}_{0}=\nabla p_{0}+\rho_{0} \nabla \Phi, \quad \mathbf{j}_{0}=\nabla \times \mathbf{B}_{0}, \quad \nabla \cdot \mathbf{B}_{0}=0,
$$

with only one $\mathrm{BC}, \mathbf{n} \cdot \mathbf{B}_{0}=0$ (at the wall).

(b) Linear perturbations $\mathbf{v}_{1}, p_{1}, \mathbf{B}_{1}, \rho_{1}$ (functions of $\mathbf{r}, t$ ) are determined by the linear PDEs

$$
\begin{aligned}
\rho_{0} \frac{\partial \mathbf{v}_{1}}{\partial t} & =-\nabla p_{1}+\mathbf{j}_{1} \times \mathbf{B}_{0}+\mathbf{j}_{0} \times \mathbf{B}_{1}-\rho_{1} \nabla \Phi, \\
\frac{\partial p_{1}}{\partial t} & =-\mathbf{v}_{1} \cdot \nabla p_{0}-\gamma p_{0} \nabla \cdot \mathbf{v}_{1}, \\
\frac{\partial \mathbf{B}_{1}}{\partial t} & =\nabla \times\left(\mathbf{v}_{1} \times \mathbf{B}_{0}\right), \quad \nabla \cdot \mathbf{B}_{1}=0, \\
\frac{\partial \rho_{1}}{\partial t} & =-\nabla \cdot\left(\rho_{0} \mathbf{v}_{1}\right),
\end{aligned}
$$

with the BCs $\mathbf{n} \cdot \mathbf{v}_{1}=0$ and $\mathbf{n} \cdot \mathbf{B}_{1}=0$ (at the wall).

An extremely effective method to reduce the system (2.2)-(2.5) is obtained by exploiting the Lagrangian displacement vector field $\boldsymbol{\xi}(\mathbf{r}, t)$, which is related to the plasma velocity by

$$
\mathbf{v}=\frac{\mathrm{D} \boldsymbol{\xi}}{\mathrm{D} t} \equiv \frac{\partial \boldsymbol{\xi}}{\partial t}+\mathbf{v} \cdot \nabla \boldsymbol{\xi} \Rightarrow \mathbf{v}_{1}=\frac{\partial \boldsymbol{\xi}}{\partial t}
$$

It permits integration of the equations (2.3)-(2.5) for $p_{1}, \mathbf{B}_{1}, \rho_{1}$, and presentation of the momentum equation (2.2) in terms of $\boldsymbol{\xi}$ alone. This yields an equation of motion (the 'Schrödinger equation') in terms of the force operator $\mathbf{F}$ :

$$
\mathbf{F}(\boldsymbol{\xi}) \equiv-\nabla \pi-\mathbf{B} \times(\nabla \times \mathbf{Q})+(\nabla \times \mathbf{B}) \times \mathbf{Q}+(\nabla \Phi) \nabla \cdot(\rho \boldsymbol{\xi})=\rho \frac{\partial^{2} \boldsymbol{\xi}}{\partial t^{2}},
$$


with the shorthand notation

$$
\pi\left(\equiv p_{1}\right)=-\gamma p \nabla \cdot \boldsymbol{\xi}-\boldsymbol{\xi} \cdot \nabla p, \quad \mathbf{Q}\left(\equiv \mathbf{B}_{1}\right)=\nabla \times(\boldsymbol{\xi} \times \mathbf{B}) .
$$

For normal modes, $\hat{\boldsymbol{\xi}}(\mathbf{r}) \mathrm{e}^{-\mathrm{i} \omega t}$, the following eigenvalue problem is obtained:

$$
\mathbf{F}(\hat{\boldsymbol{\xi}})=-\rho \omega^{2} \hat{\boldsymbol{\xi}} \Rightarrow \text { spectrum }\left\{\omega^{2}\right\} .
$$

The resulting spectrum contains both discrete and continuous parts (even on a finite interval!).

\section{Differential eqs. Quadratic forms}

('Schrödinger') ('Heisenberg')

Equation of motion: $\quad$ Hamilton's principle:

$$
\mathbf{F}(\boldsymbol{\xi})=\rho \frac{\partial^{2} \boldsymbol{\xi}}{\partial t^{2}} \quad \delta \int_{t_{1}}^{t_{2}}(K[\dot{\boldsymbol{\xi}}]-W[\boldsymbol{\xi}]) d t=0 \quad \Rightarrow \quad \begin{gathered}
\text { Full dynamics: } \\
\boldsymbol{\xi}(\mathbf{r}, t)
\end{gathered}
$$

Eigenvalue problem: Rayleigh's principle:

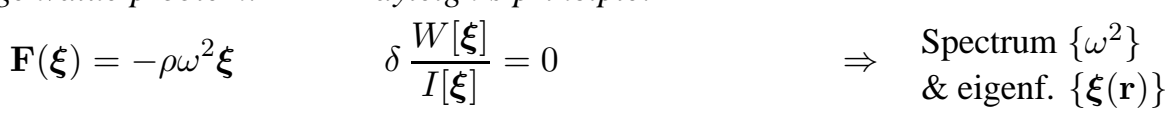

Marginal equation: $\quad$ Energy principle:

$$
\begin{aligned}
& \mathbf{F}(\boldsymbol{\xi})=0 \\
& W[\boldsymbol{\xi}]>0 \\
& \Rightarrow \quad \begin{array}{l}
\text { Stability }\left(\begin{array}{l}
\mathrm{y} \\
\mathrm{n}
\end{array}\right) \\
\& \operatorname{trial} \boldsymbol{\xi}(\mathbf{r})
\end{array}
\end{aligned}
$$

Figure 2: Two 'pictures' of MHD spectral theory

One may introduce a Hilbert space and quadratic forms for this eigenvalue problem, and thus obtain a complete (mathematical) analogy with quantum mechanics (Fig. 2):

(1) Define an inner product for vector fields $\xi$ and $\eta$ (satisfying the BCs), with finite norm,

$$
\langle\boldsymbol{\xi}, \eta\rangle \equiv \frac{1}{2} \int \rho \boldsymbol{\xi}^{*} \cdot \eta d V, \quad\|\boldsymbol{\xi}\| \equiv\langle\boldsymbol{\xi}, \boldsymbol{\xi}\rangle^{1 / 2}<\infty .
$$

(2) In this Hilbert space, the force operator $\mathbf{F}$ is a self-adjoint linear operator,

$$
\frac{1}{2} \int \eta^{*} \cdot \mathbf{F}(\boldsymbol{\xi}) d V=\frac{1}{2} \int \boldsymbol{\xi} \cdot \mathbf{F}\left(\eta^{*}\right) d V
$$

so that the eigenvalues of $\rho^{-1} \mathbf{F}$ are real. Hence, $\omega^{2} \geq 0 \Rightarrow$ stable, òr $\omega^{2}<0 \Rightarrow$ unstable.

(3) The linearized kinetic energy is related to the norm,

$$
K \equiv \frac{1}{2} \int \rho \mathbf{v}^{2} d V \approx \frac{1}{2} \int \rho \dot{\boldsymbol{\xi}}^{2} d V \equiv\|\dot{\boldsymbol{\xi}}\|^{2}<\infty .
$$

The potential energy follows from energy conservation, $\dot{W}=-\dot{K}$, and self-adjointness,

$$
W=-\frac{1}{2} \int \boldsymbol{\xi}^{*} \cdot \mathbf{F}(\boldsymbol{\xi}) d V
$$


The intuitive meaning of Eq. (2.13) for $W$ is that it is the work done against the force $\mathbf{F}$.

(4) This yields the powerful energy principle [5] to investigate stability by means of trial functions: either $W[\boldsymbol{\xi}] \geq 0$ for all $\boldsymbol{\xi} \Rightarrow$ stable, òr $W[\boldsymbol{\xi}]<0$ for a single $\boldsymbol{\xi} \Rightarrow$ unstable.

Not surprisingly, the standard spectral theory of static plasmas starts with the model problem of solving the MHD force operator equation $\mathbf{F}(\boldsymbol{\xi})=-\rho \omega^{2} \boldsymbol{\xi}$ (the time-independent 'Schrödinger equation') for a cylindrical flux tube (the ' $\mathrm{H}$ atom'). This involves first projecting the Fourier modes,

$$
\boldsymbol{\xi}(r, \theta, z)=\left(\xi_{r}(r), \xi_{\theta}(r), \xi_{z}(r)\right) \mathrm{e}^{\mathrm{i}(m \theta+k z)},
$$

onto the magnetic geometry (i.e. normal to the magnetic surfaces, $\perp$ and $\| \mathbf{B}) \Rightarrow \chi\left(\equiv r \xi_{r}\right), \eta, \zeta$. Next, eliminating the tangential components $\eta\left(\chi^{\prime}, \chi\right)$ and $\zeta\left(\chi^{\prime}, \chi\right)$, which leads to a radial wave equation for $\chi[6,7]$,

$$
\frac{d}{d r}\left(\frac{N}{r D} \frac{d \chi}{d r}\right)+\left[U+\frac{V}{D}+\left(\frac{W}{D}\right)^{\prime}\right] \chi=0
$$

where $N, D, U, V, W$ are functions of $r$ (involving the background equilibrium solutions $\rho(r), p(r)$, $\mathbf{B}(r)$ of Eq. (2.1)) and of $\omega^{2}$. Here, two kinds of singularities occur, associated with the zeros of $N$ and $D$ :

$$
\begin{array}{ll}
N \equiv \rho^{2}\left(\gamma p+B^{2}\right)\left(\omega^{2}-\omega_{A}^{2}\right)\left(\omega^{2}-\omega_{S}^{2}\right) & \Rightarrow \text { genuine continua }\left\{\omega_{S}^{2}\right\},\left\{\omega_{A}^{2}\right\} \\
D \equiv \rho^{2}\left(\omega^{2}-\omega_{s 0}^{2}\right)\left(\omega^{2}-\omega_{f 0}^{2}\right) & \Rightarrow \text { apparent continua }\left\{\omega_{s 0}^{2}\right\},\left\{\omega_{f 0}^{2}\right\} .
\end{array}
$$

The genuine continua $[8,9]$ correspond to localized Alfvén and fast magneto-sonic waves, represented by $\delta$-functions localized at particular magnetic surfaces, the apparent continua are just fake singularities $[10,11,12]$ representing the turning point frequencies of the slow and fast magnetosonic waves.

This leads to a schematic structure (Fig. 3) with the spectrum 'hanging' on the continua $\left\{\omega_{S}^{2}\right\}$, $\left\{\omega_{A}^{2}\right\}$ and $\left\{\omega_{F}^{2}(\equiv \infty)\right\}$, where the slow magneto-sonic $(\zeta)$, Alfvén $(\eta)$, or fast magno-tosonic $(\xi)$ modes become singular. Monotonicity of the spectrum outside the singularities could be proved by splitting the quadratic form in a 3D internal product and a 1D Sturm-Liouville type expression [13].

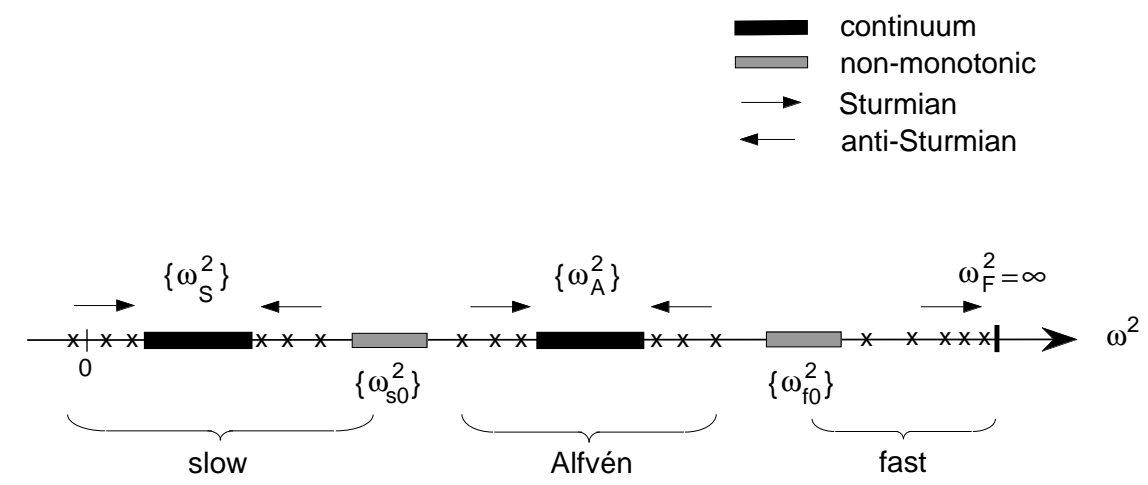

Figure 3: Schematic spectral structure of MHD waves 
The following 'picture' has emerged:

(a) MHD spectral theory is a powerful organizing principle for the dynamics of macroscopic waves and instabilities in plasmas.

(b) The spectral structure centers about the three continua $\left\{\omega_{S}^{2}\right\},\left\{\omega_{A}^{2}\right\}, \omega_{F}^{2} \equiv \infty$. For example, most of tokamak stability theory concerns 'interchange' instabilities, i.e. cluster spectra emitted by the continua when they extend to $\omega=0$ (Fig. 4):

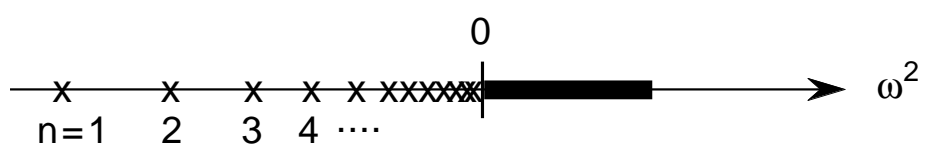

Figure 4: Local stability associated with the limits $\omega_{A} \sim \omega_{S} \sim k_{\|} B \rightarrow 0$

This is the basis of the celebrated ballooning formalism [14].

(c) The insight has been embedded in powerful numerical codes exploiting advanced eigenvalue solvers like the Jacobi-Davidson algorithm developed by Sleypen and van der Vorst [15].

(d) Folding in the data obtained from a large variety of diagnostics in tokamaks has led to the maturation of MHD spectroscopy for static laboratory plasmas.

(e) MHD spectroscopy for stationary astrophysical plasmas is still immature. Spatially resolved observations are presently absent, but they will undoubtedly emerge in 21 st century.

\section{Modifications for stationary plasmas}

For stationary plasmas, spectral theory is much more complicated than for static plasmas since the equilibrium involves the background flow field $\mathbf{v}$ :

$$
\begin{aligned}
& \nabla \cdot(\rho \mathbf{v})=0, \\
& \rho \mathbf{v} \cdot \nabla \mathbf{v}+\nabla p+\rho \nabla \Phi=\mathbf{j} \times \mathbf{B}, \quad \mathbf{j}=\nabla \times \mathbf{B}, \\
& \mathbf{v} \cdot \nabla p+\gamma p \nabla \cdot \mathbf{v}=0 \\
& \nabla \times(\mathbf{v} \times \mathbf{B})=0, \quad \nabla \cdot \mathbf{B}=0 .
\end{aligned}
$$

Hence, the equilibrium becomes a nonlinear problem in its own right.

The linear perturbations are again representable with a displacement vector field $\boldsymbol{\xi}$ [16],

$$
\begin{aligned}
\mathbf{F}(\boldsymbol{\xi})+ & 2 \mathrm{i} \rho \omega \mathbf{v} \cdot \nabla \boldsymbol{\xi}+\rho \omega^{2} \boldsymbol{\xi}=0, \\
& \mathbf{F} \equiv \mathbf{F}_{\text {static }}(\boldsymbol{\xi})+\nabla \cdot[\rho(\mathbf{v} \cdot \nabla \mathbf{v}) \boldsymbol{\xi}-\rho \mathbf{v v} \cdot \nabla \boldsymbol{\xi}],
\end{aligned}
$$

but the operator is no longer self-adjoint, so that complex eigenvalues $\omega$ occur corresponding to damped and 'overstable' modes. The MHD spectral problem,

$$
\mathbf{F}_{\text {static }}(\boldsymbol{\xi})+\nabla \cdot[\rho(\mathbf{v} \cdot \nabla \mathbf{v}) \boldsymbol{\xi}]+\rho(\omega+\mathrm{iv} \cdot \nabla)^{2} \boldsymbol{\xi}=0,
$$

is now a quadratic eigenvalue problem, involving Doppler shifts and Coriolis effects. 
The radial wave equation for the model cylindrical problem is again an ODE [17, 2],

$$
\frac{d}{d r}\left(\frac{\widetilde{N}}{r \widetilde{D}} \frac{d \chi}{d r}\right)+\left[\widetilde{U}+\frac{\widetilde{V}}{\widetilde{D}}+\left(\frac{\widetilde{W}}{\widetilde{D}}\right)^{\prime}\right] \chi=0
$$

where $\widetilde{N}, \widetilde{D}, \widetilde{U}, \widetilde{V}, \widetilde{W}$ are functions of $r$ and of the Doppler shifted frequency $\widetilde{\omega}$,

$$
\widetilde{\omega} \equiv \omega-\Omega_{0}(r), \quad \Omega_{0} \equiv m v_{\theta} / r+k v_{z} .
$$

Forward (+)/backward (-) shifted genuine and apparent continua (both real!),

$$
\begin{array}{ll}
\Omega_{S}^{ \pm} \equiv \Omega_{0} \pm \omega_{S}, & \Omega_{A}^{ \pm} \equiv \Omega_{0} \pm \omega_{A}, \quad \Omega_{F}^{ \pm} \equiv \pm \infty \\
\Omega_{s o}^{ \pm} \equiv \Omega_{0} \pm \omega_{s o}, & \Omega_{f 0}^{ \pm} \equiv \Omega_{0} \pm \omega_{f 0},
\end{array}
$$

determine the spectral structure: clustering of complex eigenvalues occurs towards the real axis.

The relationship between the MHD and hydrodynamic (HD) spectra is illustrated in the figures below, demonstrating how the latter result from taking the limit $\mathbf{B} \rightarrow 0$.

(1) Structure of the MHD spectrum, with the real slow, Alfvén, and fast continua, where (in the presence of gravity) forward and backward, Sturmian and anti-Sturmian discrete modes cluster, forming the maximum allowable number of ten sub-spectra [18]:

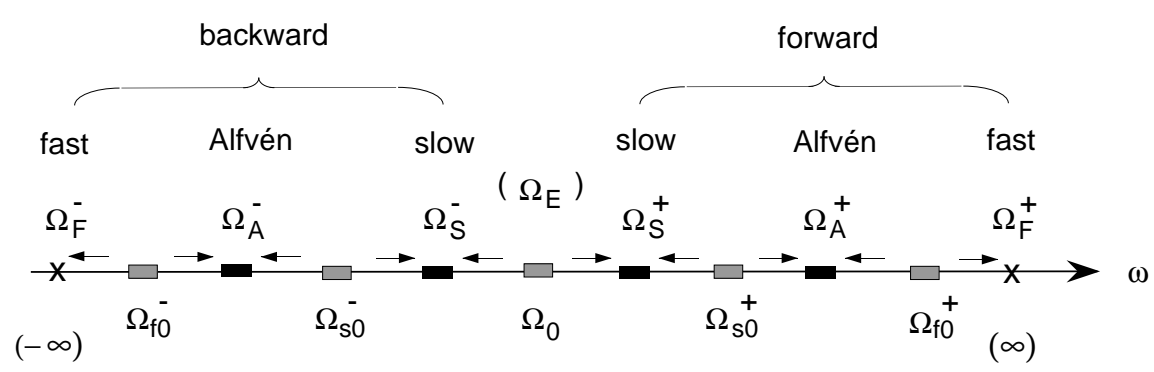

Figure 5: Schematic spectral structure of MHD waves with flow

(2) Structure of HD spectrum for ordinary shear flow fluid, with the real flow continuum (found by Case [19]), where gravity modes ( $g$-modes) cluster, whereas sound waves ( $p$-modes) cluster at $\infty$ :

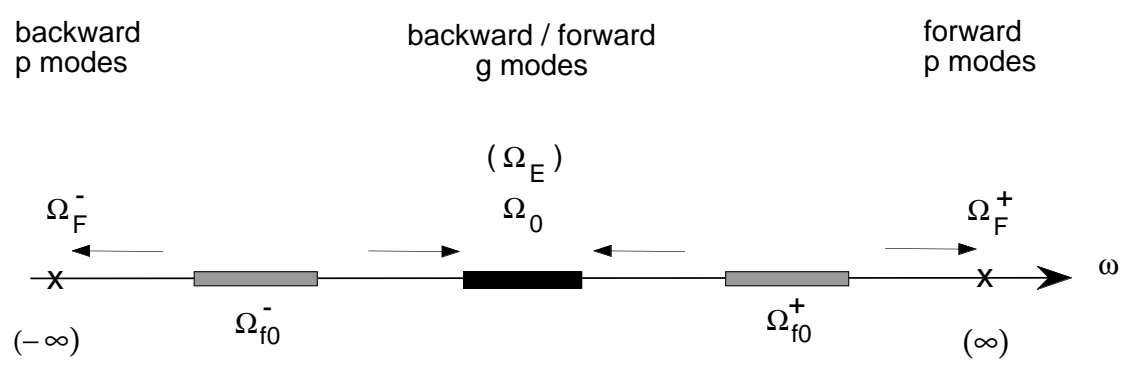

Figure 6: Schematic spectral structure of hydrodynamic waves with flow 
This analysis has produced the following new insights:

(a) In the HD limit ( $\mathbf{B} \rightarrow 0$ ), the four MHD Alfvén and slow continua collapse into the HD flow continuum:

$$
\Omega_{A}^{ \pm} \rightarrow \Omega_{0}, \quad \Omega_{S}^{ \pm} \rightarrow \Omega_{0} \quad\left(\Omega_{F}^{ \pm} \text {remains at } \pm \infty\right) .
$$

Vice versa, the flow continuum is absorbed by the four MHD continua when $B \neq 0$. Hence, in contrast to common beliefs, there is no separate flow continuum in MHD [18].

(b) For a plane plasma slab, monotonicity of the MHD spectrum along the real axis could be proved, whereas the analysis of a cylindrical plasma (with Coriolis force) required the construction of a new quadratic form $Q$ taking the place of $\omega$ in the complex plane [18].

(c) Significant progress was obtained in the understanding of the full complex spectrum (in the presence of the magneto-rotational, Rayleigh-Taylor, and Kelvin-Helmholtz instabilities) of a thin cylindrical slice as a model for accretion disks around compact objects [2, 20].

Let us now make up the balance. In astrophysical plasmas, stationary equilibrium flows must appear center stage. Hence, the complications associated with non-selfadjoint operators, Doppler shifts, eigenvalues on unknown paths in the complex $\omega$-plane, centrifugal and Coriolis effects in curved velocity fields necessarily have to be addressed. We could trace the central spectral structure (continua and monotonicity) along the real axis, and are able to accurately compute full complex spectra. However, the big challenge is yet to come: transonic flows. This requires analysis in at least two dimensions since hyperbolicity and shocks break the symmetry of the simpler 1D equilibria. Hence, our next subject is MHD spectral theory of 2D transonic flows. This is tied to the following physical problem: In accretion disks around neutron stars or black holes, accretion-ejection requires anomalous dissipation, i.e. small-scale instabilities. The standard magneto-rotational instability (MRI) only operates for low magnetic field strength. Are there instabilities that operate at arbitrary field strength?

\section{Instabilities of transonic flows}

Accretion disks rotating around massive objects, together with emitted jets, abound in astrophysics. They occur in the wide range about young stellar object (YSOs), with a mass of the order of the solar mass $\left(M_{*} \sim 1 M_{\odot}\right)$, but also about active galactic nuclei (AGNs), with the mass of hundreds of millions of stars $\left(M_{*} \sim 10^{8} M_{\odot}\right)$. They are observed with optical and radio telescopes and generally show near-relativistic jets, emitted perpendicular to the rotational plane, with a surprising stability. The detailed mechanisms of both accretion and ejection are not quite understood although the magneto-rotational instability, operating at small magnetic field strengths, is claimed to be responsible for the anomalous dissipation needed for accretion [21, 22].

Here, we enlarge the discussion to the plasma dynamics in transonically rotating magnetized (thick) disks about a compact object for arbitrary magnitude of the magnetic field. In this manner, we are able to describe the dynamics of both astrophysical objects, like accretion disks, and laboratory fusion devices, like tokamaks, from the single point of view of the magnetohydrodynamics of axi-symmetric magnetically confined plasmas. Of course, the only reason this can be done is the scale-independence of MHD discussed in Sec. 1. Fig. 7 shows the schematic configuration: a 
'superposition' of a tokamak and a black hole. The disk is inflated to a thick torus with magnetic field components in the toroidal as well as the poloidal direction (indicated by the corresponding Alfvén speeds $b_{\varphi}$ and $b_{\mathrm{p}}$ ) and also rotating in those directions (indicated by the flow speeds $v_{\varphi}$ and $v_{\mathrm{p}}$ ). In the center of the torus, a compact object of mass $M_{*}$ is placed, indicated by the symbol $g$. Of course, in laboratory devices, this object is absent. In the analysis, switching off this effect is simply effected by putting the gravitational parameter $\Gamma$ (introduced below) equal to zero.

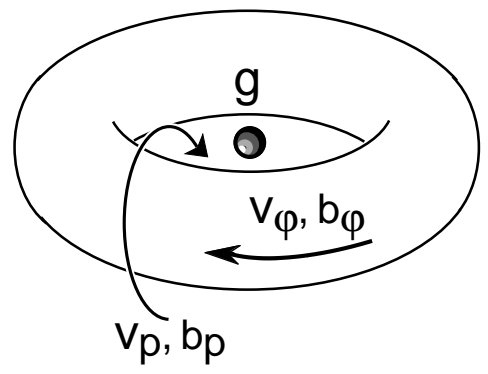

Figure 7: Rotating magnetized disk about a compact object

We investigate the stationary 2D equilibrium and the local instabilities of this configuration, assuming that the accretion speed is much smaller than the rotation speeds of the disk. We will find new instabilities driven by the transonic transitions of the flow that involve singular transslow Alfvén modes with a continuous spectrum that 'live' on the curved two- dimensional surfaces spanned by plasma velocity and magnetic field. The gravitational parameter, measuring the deviation from Keplerian flow (where $\Gamma=1$ ) is defined by

$$
\Gamma(\psi) \equiv \frac{\rho G M_{*}}{R_{0} M^{2} B^{2}} \quad\left[\approx \frac{G M_{*}}{R v_{\varphi}^{2}} \text { for parallel flow }\right]
$$

where $\psi$ is the magnetic flux (serving as a radial label), $R_{0}$ is the distance from the symmetry axis to the magnetic axis (located somewhere in the interior of the torus), and $M$ is the poloidal Alfvén Mach number (the Mach number of the poloidal flow measured with respect to the Alfvén speed). The analysis, taken from Ref. [3], is amplified by the results from two numerical codes, viz. FINESSE, for the computation of transonic equilibria [23], and PHOENIX, for the computation of the corresponding transonic MHD spectra [24].

First, the system of PDEs (3.1)-(3.4) is transformed into a variational principle for stationary MHD equilibria involving two fields, viz. the poloidal flux, $\psi$, and the square of the poloidal Alfvén Mach number, $M^{2} \equiv \rho v_{p}^{2} / B_{p}^{2}$. The stationary axi-symmetric states are then obtained by minimizing a Lagrangian,

$$
\delta \int \mathscr{L} d V=0, \quad \mathscr{L} \equiv \frac{1}{2 R^{2}}\left(1-M^{2}\right)|\nabla \psi|^{2}-\frac{\Pi_{1}}{M^{2}}-\frac{\Pi_{2}}{\gamma M^{2 \gamma}}+\frac{\Pi_{3}}{1-M^{2}},
$$

where three nonlinear composites $\Pi_{j}\left(\Lambda_{i}(\psi) ; R, Z\right)$ enter that are functions of five arbitrary flux functions $\Lambda_{i}(\psi)$. These are the stream function $\chi$, the Bernoulli function $H$, the entropy $\mathrm{S}$, the vorticity-current density stream function $K$, and the electric potential $\Phi$, which in turn are nonlinear composites of the primitive variables $\rho, p, v_{p}, v_{\varphi}, B_{p}$, and $B_{\varphi}$. 
The Euler-Lagrange equations of the variational principle (4.2) constitute a second order PDE for the flux $\psi(R, Z)$, and an algebraic equation for the square $M^{2}(R, Z)$ of the poloidal Alfvén Mach number. Substitution of the solution $M^{2}=M^{2}(|\nabla \psi|, \psi ; R, Z)$ of the latter equation back into the PDE for $\psi$ provides a rather complicated expression for the coefficients of the highest derivatives, determining the characteristics $d x^{\prime} / d y^{\prime}= \pm \sqrt{\Delta}$, where

$$
\Delta=\frac{\gamma p+B^{2}}{B_{p}^{2}} \frac{M^{2}-M_{c}^{2}}{\left(M^{2}-M_{s}^{2}\right)\left(M^{2}-M_{f}^{2}\right)} \quad\left\{\begin{array}{l}
<0: \text { elliptic } \\
\geq 0 \text { : hyperbolic }
\end{array} .\right.
$$

Here, the quantities $M_{c}, M_{s}$, and $M_{f}$ are nonlinear expressions of the primitive equilibrium variables that yield two hyperbolic flow regimes, viz. a slow regime for $M_{c}^{2}<M^{2}<M_{s}^{2}$ and a fast regime for $M^{2}>M_{f}^{2}$. For other values of $M^{2}$ the flow is either elliptic or forbidden (existence of solutions is not guaranteed for all arbitrary choices of the five flux functions).

We then arrive at what may be called the transonic enigma:

(1) At transonic transitions, the character of the equilibrium flow changes from elliptic to hyperbolic. Consequently, standard tokamak equilibrium solvers diverge in the hyperbolic regimes. For the time being, the 'remedy' appears to be to restrict the calculation to the elliptic regimes which in MHD (in contrast to ordinary hydrodynamics) contain both sub- and super-critical regimes.

(2) Because of the transonic transitions, the linear waves (associated with time dependence) and the nonlinear stationary states (associated with the 2D spatial dependence) become interconnected, as illustrated by the figures below:

- The wave spectra cluster at the continuous spectra $\left\{ \pm \omega_{S}\right\},\left\{ \pm \omega_{A}\right\}, \pm \infty\left(\omega_{F}\right)$, for simplicity here shown for the static equilibrium case:

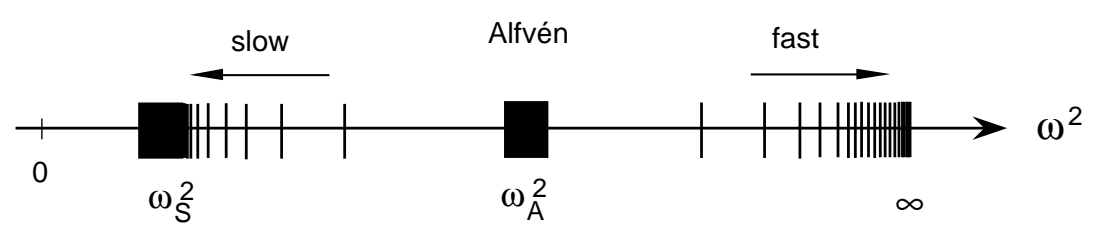

Figure 8: Asymptotic behavior of the three MHD waves

- The hyperbolic flow regimes are delimited by critical values of the poloidal Alfvén Mach number that correspond to the above singularities, where it is to be noted that the hyperbolic Alfvén regime consists of a singularly isolated point in the middle of a forbidden flow regime [25]:

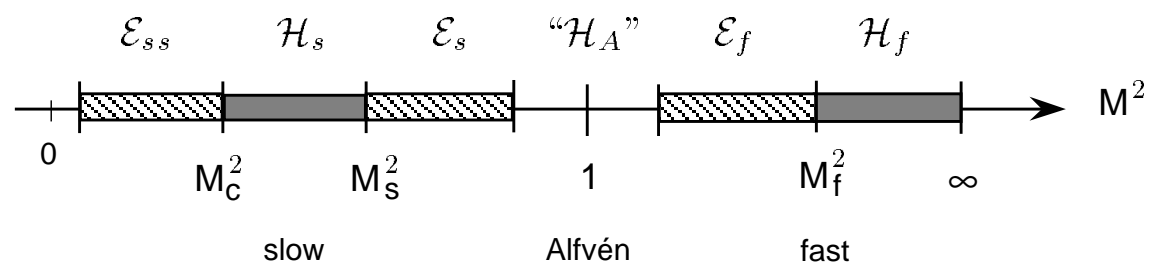

Figure 9: Corresponding three hyperbolic MHD flow regimes 
It is of interest to investigate the stability properties in the first elliptic flow regime (indicated by $\mathscr{E}_{S}$ in Fig. 9). In this regime, we studied transonic continuum modes, i.e. singular modes localized about a single magnetic/flow surface and mainly directed tangential to it:

$$
\begin{aligned}
& \eta(\psi, \vartheta, \varphi) \approx \delta\left(\psi-\psi_{0}\right) \hat{\eta}(\vartheta) \mathrm{e}^{\mathrm{i} n \varphi} \\
& \zeta(\psi, \vartheta, \varphi) \approx \delta\left(\psi-\psi_{0}\right) \hat{\zeta}(\vartheta) \mathrm{e}^{\mathrm{i} n \varphi}
\end{aligned}
$$

For each magnetic surface, labeled by $\psi_{0}$, we then obtain a discrete eigenvalue problem,

$$
\begin{gathered}
\widehat{\mathbf{A}} \equiv\left(\begin{array}{cc}
\mathscr{A} \cdot \widehat{\mathbf{V}}=\widehat{\mathbf{B}} \cdot \widehat{\mathbf{V}}, & \widehat{\mathbf{V}} \equiv(\hat{\eta}, \hat{\zeta})^{\mathrm{T}} \\
B^{2} & \mathscr{F}-\left(M^{2}-M_{c}^{2}\right) \frac{B^{2}}{\rho^{2}}\left[\partial\left(\frac{\rho R B_{\varphi}}{B^{2}}\right)\right]^{2}-\mathrm{i}\left(M^{2}-M_{c}^{2}\right) \frac{B^{2}}{\rho^{2}}\left[\partial\left(\frac{\rho R B_{\varphi}}{B^{2}}\right)\right] \mathscr{F} \rho \\
\mathrm{i} \rho \mathscr{F}\left(M^{2}-M_{c}^{2}\right) \frac{B^{2}}{\rho^{2}}\left[\partial\left(\frac{\rho R B_{\varphi}}{B^{2}}\right)\right] & \mathscr{F} M_{c}^{2} B^{2} \mathscr{F}+\rho\left[\partial\left(\left(M^{2}-M_{c}^{2}\right) \frac{B^{2}}{\rho^{2}} \partial \rho\right)\right]
\end{array}\right), \\
\widehat{\mathbf{B}} \equiv\left(\begin{array}{cc}
(\sqrt{\rho} \widetilde{\omega}-\mathscr{F} M) \frac{R^{2} B_{p}^{2}}{B^{2}}(\sqrt{\rho} \widetilde{\omega}-M \mathscr{F}) & -\mathrm{i} \alpha \sqrt{\rho} \widetilde{\omega} \\
\mathrm{i} \alpha \sqrt{\rho} \widetilde{\omega} & (\sqrt{\rho} \widetilde{\omega}-\mathscr{F} M) B^{2}(\sqrt{\rho} \widetilde{\omega}-M \mathscr{F})
\end{array}\right),
\end{gathered}
$$

where $\widetilde{\omega} \equiv \omega-n \Omega$ is the Doppler shifted improper eigenfrequency in a frame rotating with the angular velocity $\Omega, \alpha$ is the Coriolis parameter, $[\partial(\ldots)]$ indicates derivatives of the equilibrium quantities tangential to the magnetic surfaces, and $\mathscr{F}$ is the derivative along the magnetic field lines. The continuous spectrum is mapped out by the collection of solutions of Eq. (4.5) for all different values of $\psi$ (ranging from 0 on the magnetic axis to 1 at the wall).

Note that we have a truly localized problem now, associated with modes that are 'living' on the two-dimensional surfaces spanned by the magnetic field lines and coinciding with the surfaces of the flow. The terms on the diagonal of the matrix $\widehat{\mathbf{A}}$ suggest that those modes of the continuous spectrum become unstable in the trans-slow $\left(M^{2}>M_{c}^{2}\right)$ elliptic flow regime. These modes could be studied in accurate detail by means of the newly developed dual set of equilibrium-stability codes FINESSE-PHOENIX [23, 24]. One particular result is shown in Fig. 10 where the continuous spectrum is plotted in the complex $\omega$-plane for a thick accretion disk (torus) with flow parallel to the magnetic field lines and the value $\Gamma=2$ for the gravitational parameter. The colors indicate the radial position $s \equiv \sqrt{\psi}$ of the modes. Some of the continuum modes are located on the real $\omega$-axis, as in static plasmas, but the flow now drives a significant part of them into the complex plane to become unstable $(\operatorname{Im} \omega>0)$. Typically, two real modes coalesce and then produce a pair of two complex conjugate modes, one of which is an 'overstable' mode (a wave with an exponentially growing amplitude). Overall, two kinds of overstable modes occur, corresponding to modes propagating clockwise or anti-clockwise in the poloidal direction (the short way around the torus). For the parameters chosen, these pairs of modes interact to form locked modes ( $\operatorname{Re} \omega=0$ ) with huge exponential growth rates, on the scale of the rotation period of the disk, shown in the middle of the figure. 


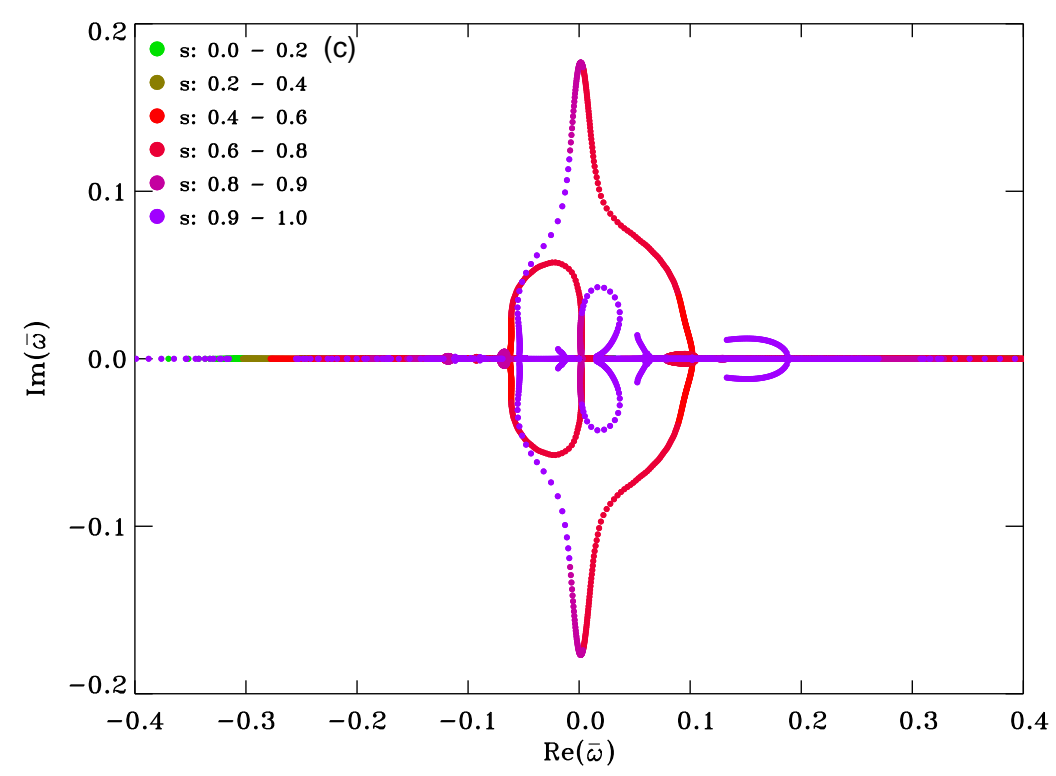

Figure 10: Complex trans-slow continuum instabilities, $\Gamma=2, n=1$

Led by the numerical results, which exhibit dominant coupling of six slow magneto-sonic and Alfvén continuum modes, $S_{m-1}^{ \pm}, A_{m}^{ \pm}, S_{m+1}^{ \pm}$, which are degenerate at the rational magnetic surfaces (where $q=-m / n$ ), an explicit analysis of the dispersion equation could be carried out by small inverse aspect ratio expansion. This confirms that the trans-slow Alfvénic continuum modes are unstable at, or close to, the rational surfaces for all toroidal mode numbers $n$. For a very massive central object $(\Gamma \gg 1)$, the growth rate in the limit $|n|,|m| \rightarrow \infty$ becomes huge,

$$
\bar{\omega} \approx(\mathrm{i} / \sqrt{2 \rho}) M \Gamma,
$$

far in excess of the Alfvén frequency. Since these modes are localized both radially and in the angles $\vartheta$ and $\varphi$, they are perfectly suitable to produce turbulence of the flow needed to produce both accretion and detachment of the flow from the magnetic field involved in jet formation.

\section{Conclusioning remarks}

(a) MHD spectroscopy of extended magnetic flux tubes describes traveling Alfvén wave excitations of magnetic strings that can be observed everywhere in the Universe.

(b) In transonic plasma flows about compact objects, the continuous spectra of those waves are explosively unstable, facilitating both accretion and ejection of jets from accretion disks about those objects.

(c) It is appropriate to close with expressing deep appreciation of the mathematical power of Hilbert space (1912), that first provided the mathematical apparatus for quantum mechanics and now also for the completely classical theory of magnetohydodynamics. With the many space missions planned to provide spatial resolutions of a large variety of astrophysical objects, and knowing that most of them are dominated by plasma dynamics, MHD spectroscopy awaits a great future! 


\section{Appendix: Variational principle for the stationary states of two-fluid plasmas}

For the stationary axi-symmetric equilibrium states of two-fluid plasmas, a similar variational principle as Eq. (4.2) could be derived [26]:

$$
\begin{gathered}
\delta \int \mathscr{L}_{\mathrm{TF}}\left(\chi_{\alpha}, \nabla \chi_{\alpha}, \rho_{\alpha}, \psi, \nabla \psi, \widetilde{\phi}, \nabla \widetilde{\phi}, \widetilde{\mathscr{V}}, \nabla \widetilde{\mathscr{V}} ; R, Z\right) d V=0 \quad(\alpha=e, i), \\
\mathscr{L}_{\mathrm{TF}} \equiv \frac{1}{2 \rho_{e} R^{2}}\left|\nabla \chi_{e}\right|^{2}+\frac{1}{2 \rho_{i} R^{2}}\left|\nabla \chi_{i}\right|^{2}-\frac{1}{2 \mu_{0} R^{2}}|\nabla \psi|^{2}+\frac{1}{2} \varepsilon_{0}|\nabla \widetilde{\phi}|^{2}-\frac{1}{8 \pi G}|\nabla \widetilde{\mathscr{V}}|^{2} \\
+\frac{1}{2 \mu_{0} R^{2}} I^{2}+\rho_{e} F_{e}-\frac{1}{\gamma-1} \rho_{e}{ }^{\gamma} S_{e}+\rho_{i} F_{i}-\frac{1}{\gamma-1} \rho_{i}^{\gamma} S_{i} .
\end{gathered}
$$

It involves the seven fields $\chi_{e}, \chi_{i}, \rho_{e}, \rho_{i}, \psi, \phi, \mathscr{V}$, i.e. the electron and ion stream function for the poloidal flow, the electron and ion density, the poloidal magnetic flux, the electric potential, and the gravitational potential, respectively, and three nonlinear composites of those fields,

$$
\begin{gathered}
I\left(\chi_{e}, \chi_{i}\right) \equiv R B_{\varphi}=I_{0}+\mu_{0}\left[\left(e / m_{e}\right) \chi_{e}-\left(Z e / m_{i}\right) \chi_{i}\right], \\
F_{e}\left(\chi_{e}, \psi, \widetilde{\phi}, \widetilde{\mathscr{V}} ; R, Z\right) \equiv H_{e}-\frac{1}{2 R^{2}}\left(L_{e}-\frac{e}{m_{e}} \psi\right)^{2}+\frac{e}{m_{e}}\left(\phi_{*}+\widetilde{\phi}\right)-\mathscr{V}_{*}-\widetilde{\mathscr{V}}, \\
F_{i}\left(\chi_{i}, \psi, \widetilde{\phi}, \widetilde{\mathscr{V}} ; R, Z\right) \equiv H_{i}-\frac{1}{2 R^{2}}\left(L_{i}+\frac{Z e}{m_{i}} \psi\right)^{2}-\frac{Z e}{m_{i}}\left(\phi_{*}+\widetilde{\phi}\right)-\mathscr{V}_{*}-\widetilde{\mathscr{V}},
\end{gathered}
$$

which in turn involve six arbitrary stream functions $H_{e, i}\left(\chi_{e, i}\right), L_{e, i}\left(\chi_{e, i}\right), S_{e, i}\left(\chi_{e, i}\right)$ and the three potentials $\psi, \phi, \mathscr{V}$. Here. $H_{e, i}$ are the Bernoulli functions, $L_{e, i}$ are the generalized specific angular momenta, and $S_{e, i}$ are the specific entropies of the electrons and ions. Needless to say, this problem is considerably more complex than the ideal MHD stationary equilibria, since it also contains the microscopic scales of the electromagnetic, plasma and cyclotron waves, so that the scale-independence of ideal MHD is lost. Here, transonic transitions also occur, when the electron and ion flow speeds surpass the respective sound speeds, corresponding to the small-scale asymptotics of the waves of the two-fluid plasma. The associated transonic enigma and its implications for the calculation of the spectrum of two-fluid waves and instabilities has been indicated, but not investigated in any detail.

\section{References}

[1] J.P. Goedbloed and S. Poedts, Principles of Magnetohydrodynamics (Cambridge University Press, 2004); ISBN 0521626072. http://www.cambridge.org/uk/catalogue/catalogue.asp?isbn=0521626072

[2] R. Keppens, F. Casse, and J.P. Goedbloed, 'Waves and instabilities in accretion disks: Magnetohydrodynamic spectroscopic analysis', Astrophys. J. 569 (2002), L121-L126.

[3] J.P. Goedbloed, A.J.C. Beliën, B. van der Holst, and R. Keppens, 'Unstable continuous spectra of transonic axi-symmetric plasmas', Phys. Plasmas 11 (2004), 28-54.

[4] J.P. Goedbloed, G.T.A. Huysmans, H. Holties, W. Kerner and S. Poedts, 'MHD spectroscopy: Free boundary modes (ELM's) and external excitation of TAE modes', Plasma Phys. Contr. Fusion 35 (1993), B277-292. 
[5] I. B. Bernstein, E. A. Frieman, M. D. Kruskal and R. M. Kulsrud, 'An energy principle for hydromagnetic stability problems', Proc. Roy. Soc. (London) A244 (1958), 17-40.

[6] K. Hain and R. Lüst, 'Zur Stabilität zylinder-symmetrischer Plasmakonfigurationen mit Volumenströmen', Z. Naturforsch. 13a (1958), 936-940.

[7] J. P. Goedbloed, 'Stabilization of magnetohydrodynamic instabilities by force-free magnetic fields II. Linear pinch', Physica 53, 501-534 (1971).

[8] C. Uberoi, 'Alfvén waves in inhomogeneous magnetic fields', Phys. Fluids 15 (1972), 1673-1675.

[9] H. Grad, 'Magnetofluid-dynamic spectrum and low shear stability', Proc. Natl. Acad. Sci. USA 70 (1973) 3377-3281.

[10] K. Appert, R. Gruber and J. Vaclavik, 'Continuous spectra of a cylindrical magnetohydrodynamic equilibrium’, Phys. Fluids 17 (1974), 1471-1472.

[11] J.M. Greene, private communication (1974).

[12] J.P. Goedbloed, 'Once more: The continuous spectrum of ideal magnetohydrodynamics', Phys. Plasmas 5 (1998), 3143-3154.

[13] J. P. Goedbloed and P. H. Sakanaka, 'New approach to magnetohydrodynamic stability. I. A practical stability concept', Phys. Fluids 17 (1974), 908-918.

[14] J. W. Connor, R. J. Hastie and J. B. Taylor, 'High mode number stability of an axi symmetric toroidal plasma', Proc. Roy. Soc. (London) A365 (1979), 1-17.

[15] G. L. G. Sleijpen and H. A. van der Vorst, 'A Jacobi-Davidson iteration method for linear eigenvalue problems, SIAM J. Matrix Anal. Appl. 17 (1996), 401-425.

[16] E. Frieman and M. Rotenberg, 'On the hydromagnetic stability of stationary equilibria', Rev. Modern Physics 32 (1960), 898-902.

[17] A. Bondeson, R. Iacono and A. H. Bhattacharjee, 'Local magnetohydrodynamic instabilities of cylindrical plasma with sheared equilibrium flows', Phys. Fluids 30 (1987), 2167-2180.

[18] J. P. Goedbloed, A. J. C. Beliën, B. van der Holst and R. Keppens, 'No additional flow continua in magnetohydrodynamics', Phys. Plasmas 11 (2004), 4332-4340.

[19] K. M. Case, 'Stability of inviscid plane Couette flow', Phys. Fluids 3 (1960), 143-148.

[20] J.W.S. Blokland, E. van der Swaluw, R. Keppens and J.P. Goedbloed, 'Magneto-rotational overstability in accretion disks', Astron. Astrophys. 444, 337-346 (2005).

[21] S. A. Balbus and J. F. Hawley, 'A powerful local shear instability in weakly magnetized disks. I. Linear analysis', Astrophys. J. 376 (1991), 214-222.

[22] S. A. Balbus and J. F. Hawley, 'Instability, turbulence, and enhanced transport in accre tion disks', Rev. Modern Physics 70 (1998), 1-53.

[23] A.J.C. Beliën, M.A. Botchev, J.P. Goedbloed, B. van der Holst and R. Keppens, 'New numerical tools to study waves and instabilities of flowing plasmas', Comp. Phys. Comm. 147 (2002), 497-500.

[24] J.W.S. Blokland, B. van der Holst, R. Keppens and J.P. Goedbloed, 'PHOENIX: MHD spectral code for rotating laboratory and gravitating astrophysical plasmas’, to appear (2007).

[25] J.P. Goedbloed and A. Lifschitz, 'Stationary symmetric magnetohydrodynamic flows', Phys. Plasmas 4 (1997), 3544-3564.

[26] J.P. Goedbloed, 'Variational principles for stationary one- and two-fluid equilibria of axi- symmetric laboratory and astrophysical plasmas’, Phys. Plasmas, 11 (2004), L81-L84. 